to check it. In fact, not only is the reasoning on which it is established evidently wrong, but, should it be true, it would lead to the amusing conclusion that the geodesics of any surface whatever belong to spaces of three dimensions! This mistake is of no little moment: it invalidates many results in different sections of the book.

Section II opens with the following question: Is it possible to find a lower limit to the dimensionality of the Euclidean space (plenary homaloid) containing a surface with a given arc-element? The answer, given by Darboux in 1872, and referred to in all standard treatises on the subject (like Darboux's and Bianchi's) is in the affirmative. It is always possible to construct such a surface in a space of three dimensions by integrating a MongeAmpere equation and by quadratures. Prof. Forsyth's answer is in the negative*: it is true that he speaks of an upper limit, but what he means (as can be seen from the discussion) is a lower limit. This is all the more reason that a search for an upper limit has no value, it being evident that, given a surface in a 3 -space, surfaces with the same arc-element can be found in a space with greater number of dimensions. Prof. Forsyth seems struck by the example he gives of a surface in a space with an infinite number of dimensions : there is nothing surprising in it, the dimensionality of the space having nothing to do with the arcelement of the surface (it is not a Riemannian character).

Another topic to be mentioned-and this will be the last one-is the statement, often repeated and invalidating many results, that when a certain determinant vanishes (see, for example, vol. 1, p. 369) or, what is the same thing, when the first and second derivatives of the co-ordinates of a point of a surface are not linearly independent,

* The correct result is given in a corrigenda sheet which has been issued since publication of the book. the surface lies in a quadruple homaloid. To make the mistake more curious, the example given before of a surface belonging to a space with infinite dimensions contradicts the last statement. But this is only one example, and the simplest one, of the contradictions which arise when the projective characters of the manifold are not considered. I have emphasized many times the fact that, when studying the differential properties of a given order (of differentiation), the basic element to be considered is not the dimensionality of the space in which the manifold is immersed (which may also be infinite, with no danger) but the dimensionality of certain osculating spaces (as I call them) projectively connected with the manifold.

The author describes his book as an adventure into the realm of investigating manifolds (not curves or hypersurfaces) existing in a plenary space. Thirty years ago, E. E. Levi (who is not quoted in this work) entered the same realm, harvesting the first results (for two-dimensional surfaces) and showing its difficulties. More than twenty years ago, I tackled the problem again, and conceived the idea of Riemannian geometries of higher species ("Geometrie Riemanniane di specie superiore”. Mem. R. Accad. d'Italia, 1935), showing that the differential properties of a given order $r$ of a manifold (whatever its embedding space may be) can be described in terms of $r$ differential forms of the first order and of $2,4, \ldots, 2 r$ degree. But the step was not easy. To fill the gap it was necessary to build up the projective differential geometry of manifolds, or more exactly of linear partial differential equations. In the meantime, other investigators have taken part in the work, as Vitali, Enea Bortolotti, Burstin, Duschek and Meyer; apparently passing over all this work, Prof. Forsyth has followed his own way. E. Bompiani.

\title{
Sea Urchins
}

\section{A Monograph of the Echinoidea}

2 : Bothriocidaroida, Melonechinoida, Lepidocentroida and Stirodonta. By Th. Mortensen. Text. Pp. vi +647 . Atlas. Pp. ii $+16+89$ plates. (Copenhagen : C. A. Reitzel; London : Oxford University Press, 1935.) 2 vols., 140s. net.

$\mathrm{T}$ HIS monograph attracts a review, for it exhibits features of a distinctly novel nature and it approaches perfection as near as can be. The animals are the box-like sea-urchins, starfishes with radiating segments and brick-like walls (tests) decorated with spines and fine sculpturing. They walk by outpushings of fine tubes (tube-feet) with terminal sucking-disks arranged in five rays, such as cause starfish to be of unique personality. They belong to Mortensen more than to anyone else, for he has pursued the living forms in most parts of the world. Here, his study of the urchins' habits, food and ecology was antecedent to that of their classification, and he thus by analogy can tell us much of the life of the extinct forms. Of the latter there is a long series from the Palæozoic, and it would seem 
probable that these animals have never been less numerous either in genera and species or in individuals than they are to-day. Thus the relatively deep-living Salenidæ had eighty-five species in the Cretaceous as compared with only twelve species to-day, but all the extinct forms were not deep-living.

The first animal here considered, Bothriocidaris, was formerly regarded as the ancestor of all seaurchins, but more ancient forms are now known, and Mortensen evidently places it here to avoid making another phylum. He supposes that it must have ingested food through the mouth, probably the adoral spines and tube-feet helping as in the living spatangoids. Judging by the deposits where it occurs, it lived in shallow reef-pools, which, according to the indications furnished by its test, cannot have been exposed to strong waveaction. It is this thought of the fossil forms as once alive and playing their parts on the ocean floor which makes this monograph so illuminating, its animals far more than mere dates in a strati- graphical series and its living forms the few survivors of a long ancestry.

Only three divisions (Melonechinoida, Lepidocentroida and Stirodonta), besides Bothriocidaroida with its single genus, are monographed. They contain 22 recent and 116 fossil genera. All the genera are described, while in addition the 84 existing species are fully considered in respect to their anatomy, colour, occurrence and, so far as possible, their ecology; the text illustrations of the anatomy form a feature of great utility. The same technique is applied to the fossil genera, the anatomy of which is illustrated while their affinities are considered. Many of the species are referred to, but it is beyond the author's scope to consider all the described fossil forms. Added to the monograph is a volume of plates of high quality.

The whole is of great credit not only to Mortensen but also to Denmark and to the managers of the Carlsberg Fund, whose financial aid alone made possible the printing of this luxurious publication, which it is a joy to handle.

J. S. G.

\section{Negroes and Pygmies}

\section{My Pygmy and Negro Hosts}

By Paul Schebesta. Translated from the German by Gerald Griffin. Pp. $287+31$ plates. (London : Hutchinson and Co. (Publishers), Ltd., 1936.) 18s. net.

$\mathrm{H}^{\mathrm{A}}$ THER PAUL SCHEBESTA, whose intimate knowledge of the pygmy problem, acquired in the Far East as well as in Africa, transcends that of any living ethnologist, has now produced a sequel to "Among Congo Pigmies". In this he supplements his earlier work on the Congo pygmies by an account of the Batwa of Ruanda and the Bachwa of the Equatorial Province, who, if not all pygmies, at least merit the title of half-pygmies. More important, however, for his present purpose, are the tribes of full stature with whom he came into contact on his travels in 1929 and the two succeeding years.

Interesting for the ethnologist as must be the comparison of the material relating to the pygmy peoples given here with that of the previous book, it will probably appear to most readers that the author has displayed sound judgment in devoting the larger share of his attention to the tribes other than pygmy. For one thing, this material is essential for an understanding of the ethnological background, in view of the close relation, almost a symbiosis, between the pygmy and his taller neighbour, while in the second place, the culture of these peoples is both intrinsically interesting, and for the most part has not previously been described. Witchcraft, cannibalism and tribal history afford the author a rich field for description. It may be hoped that material for a fuller and more systematic analysis of the last named will be forthcoming later. Some further details, too, of the evidence upon which is based the suggestion of a surviving influence from Ancient Egypt will be welcomed.

The author was much impressed by his visit to the Royal Court of the intrusive Batutsi of the mandated territory of Ruanda, in which, as he points out, there are certain close resemblances to the court of Uganda. He was admitted to the presence of the Royal Mother-a privilege formerly denied to the foreigner, and evidently resented. Shortly after, the dynasty was deposed by the Belgian authorities.

The narrative offers innumerable tempting paths which would lead to discussion, such as, for example, the association of initiation ceremonial with a cannibalistic 'leopard' society among the Babali, where also initiation takes the place of circum. cision, universal among the other tribes. The author himself was responsible for the discovery of a secret 'leopard men' society and its association with cannibalism among the Babali. 